Service social

\title{
La périnatalité au Québec : Une série d'études significatives à plusieurs égards
}

\author{
Michel O’Neill, Francine Saillant, Louise Pelletier, Denyse Leclerc et Linda \\ Lepage
}

Volume 39, numéro 2, 1990

Les problèmes sociaux

URI : https://id.erudit.org/iderudit/706486ar

DOI : https://doi.org/10.7202/706486ar

Aller au sommaire du numéro

Éditeur(s)

École de service social de l'Université Laval

ISSN

1708-1734 (numérique)

Découvrir la revue

Citer cet article

O’Neill, M., Saillant, F., Pelletier, L., Leclerc, D. \& Lepage, L. (1990). La périnatalité au Québec : Une série d'études significatives à plusieurs égards. Service social, 39(2), 217-234. https://doi.org/10.7202/706486ar

\section{Résumé de l'article}

Dans un premier temps, cet article présente brièvement le contexte historique des services périnatals au Québec, en particulier depuis les années 1970 où ce contexte s'est assez profondément modifié. Il passe ensuite en revue les cinq études qui composent la série La périnatalité au Québec et se termine par quelques constats d'ensemble sur ces ouvrages quant à leur valeur intrinsèque et à leur signification dans l'histoire des services périnatals québécois. 


\title{
La périnatalité au Québec Une série d'études significatives à plusieurs égards
}

\author{
Michel O'Neill \\ Francine Saillant \\ Louise Pelletier \\ Denyse Leclerc \\ Linda Lepage
}

La publication en 1989 des cinq volumes de la série La périnatalité au Québec peut être regardée sous au moins deux angles. D'une part, il s'agit là d'un ensemble d'informations cohérentes et complémentaires dont on peut discuter de l'utilité et de la pertinence pour la population et pour les praticiens et praticiennes dans le domaine des services sociaux et de santé. D'autre part, la publication de ces cinq ouvrages constitue un épisode dans l'histoire des pratiques périnatales au Québec, histoire passionnante et passionnée s'il en est, où divers protagonistes s'affrontent depuis près d'une vingtaine $d^{\prime}$ 'années avec des visions et des approches parfois complémentaires mais souvent opposées quant aux manières de prendre en charge la grossesse, l'accouchement et les premières années de la vie.

Dans les pages qui suivent, nous allons donc analyser sous ces deux angles la publication de cette série d'études. Dans un premier temps, nous camperons brièvement le contexte historique des services périnatals au Québec, en particulier depuis les années 1970 où ce contexte s'est assez profondément modifié. Dans un second temps, nous passerons en revue les cinq livres de la série pour conclure avec quelques constats d'ensemble sur ces ouvrages quant à leur valeur intrinsèque et à leur signification dans l'histoire des services périnatals québécois.

\section{Quelques éléments de contexte}

L'histoire des pratiques périnatales au Québec, depuis les débuts de la Nouvelle-France jusque vers 1950 environ, peut se résumer rapidement. Jusqu'au début du XXe siècle, autant dans la société traditionnelle blanche que dans les sociétés autochtones, l'accouchement et les relevailles étaient pris en charge par la famille élargie et le voisinage, généralement avec l'aide d'une sage-femme et avec une intervention professionnelle à peu près absente (Laforce, 
1987a; Routhier, 1987; Cournoyer, 1987; Dufour, 1987). Vers la fin du XIXe siècle, un groupe de médecins se vit reconnaître par l'État québécois, au détriment des autres intervenants et intervenantes, un statut professionnel et le monopole sur la pratique de certains gestes techniques qui, bientôt, incluront ceux concernant la grossesse et l'accouchement (Laforce, 1987b). La première moitié du XXe siècle vit donc, lentement mais sûrement, les médecins prendre possession du champ périnatal, en excluant de plus en plus les intervenantes traditionnelles à partir d'une rationalité scientifique et technique.

Cette "médicalisation » de la périnatalité s'est accélérée dramatiquement après la seconde guerre mondiale, avec l'avènement de la médecine spécialisée pratiquée en milieu hospitalier (Laurendeau, 1987), au point d'évacuer à peu près complètement du processus, les sages-femmes et, presque, les femmes elles-mêmes : on les « accouchait endormies " durant les années 1950 et une bonne partie des années 1960. Le processus de médicalisation commença cependant à être battu en brèche au début des années 1970 .

En 1973, le ministère de la Santé et des Services sociaux du Québec (MSSS), qui s'appelait alors le ministère des Affaires sociales (MAS) et qui venait tout juste d'être créé, lançait sa toute première politique : une politique en périnatalité. En effet, le Québec, qui venait à peine de réformer son système de santé suite aux recommandations de la Commission Castonguay-Nepveu, avait à ce moment le sort peu enviable d'être une des provinces canadiennes les plus défavorisées du point de vue de la mortalité maternelle et infantile. Cette politique (Blanchet, 1979) visait la réduction de ces deux problèmes, principalement par une série de mesures administratives (désignation de certains centres hospitaliers comme centres spécialisés pour les grossesses à "risques élevés ", fermeture des unités d'obstétrique faisant moins de 100 accouchements par an) et éducatives (mise sur pied systématique de cours prénatals sous la responsabilité du réseau des trente-deux Départements de santé communautaire).

Le fait le plus marquant des années 1970 en rapport avec la périnatalité est cependant le mouvement pour la santé des femmes qui, dans la foulée du féminisme ici comme ailleurs, s'est structuré durant cette décennie (De Koninck et al., 1981). Le rapport au corps et à la conception a été le premier dossier auquel le féminisme québécois s'est attaqué politiquement (Saillant, 1985). Les batailles pour I'humanisation des accouchements en milieu hospitalier (Savard, 1987) ainsi que la re-naissance des sages-femmes pour accompagner des femmes et des couples ayant décidé de se passer du système médical (Saillant et al., 1987), sont des phénomènes qui se sont intensifiés à mesure que cette décennie s'écoulait. 
En 1980, l'Association pour la santé publique du Québec (ASPQ), avec le support financier du MAS, suscitait un large débat autour du thème "Accoucher ou se faire accoucher". Elle organisa onze rencontres dans toutes les régions du Québec où plus de 10000 participants et participantes vinrent proposer une analyse critique de la manière dont la périnatalité était prise en charge par le système de soins (De Koninck, 1981).

Suite à ce bilan plutôt négatif, le MAS, en collaboration avec les établissements du réseau des affaires sociales, mit sur pied un comité de travail chargé de faire le point sur les services en périnatalité. Ce comité fit plusieurs démarches et proposa trois documents : des orientations en périnatalité, en 1984, précédées de deux autres documents de travail intitulés "Éléments de réponses aux recommandations des colloques Accoucher ou se faire accoucher " et "Pour une éducation sanitaire en milieux populaires dans le domaine de la périnatalité ». Finalement, en 1985, ce processus culminait en un "Projet de politique en périnatalité " (MAS, 1985) où étaient suggérées de nouvelles orientations, qui tenaient compte des acquis technologiques mis en place dans le système depuis la première politique de 1973 mais insistaient sur l'humanisation des soins et services.

Certaines des actions proposées par ce projet de politique (MAS, 1985 : 43-59), en particulier le suivi prénatal par les infirmières de Centres locaux de services communautaires (CLSC) et la mise sur pied de projets pilotes de maternités prises en charge par des sagesfemmes, furent reçues par plusieurs avec consternation, pour ne pas dire agressivité, en particulier par la profession médicale qui voyait là une attaque de plus à un monopole se faisant lentement éroder depuis le milieu des années 1970.

Le projet de 1985, trop délicat au plan politique, ne fut pas mis en œuvre. Le ministère demanda plutôt à divers groupes d'experts provenant principalement des Départements de santé communautaire (DSC) et du MSSS, de réaliser un certain nombre d'études complémentaires et de formuler des avis face à cinq thèmes considérés comme prioritaires : la pratique des sages-femmes, les grossesses chez les adolescentes, les grossesses en milieux défavorisés, l'état de la mortalité et de la morbidité périnatales et, finalement, l'impact de l'arrivée d'un nouvel enfant dans la famille. Ces cinq avis ont été préparés entre 1986 et 1989 et finalement publiés durant cette dernière année; ces ouvrages sur la périnatalité au Québec font ici l'objet de notre analyse critique. 
Francine Saillant, professeure, École des sciences infirmières, Université Laval.

La pratique des sages-femmes, par le Comité de travail sur la pratique des sages-femmes au Québec, Ministère de la Santé et des Services sociaux, Québec, 1989, 155 pages.

Le premier tome de la série La périnatalité au Québec est le produit des efforts du Comité de travail sur la pratique des sagesfemmes au Québec, mis sur pied en 1986 par la ministre de la santé et des services sociaux d'alors, madame Thérèse Lavoie-Roux. Principalement initiés en fonction du mandat « de fournir à la Ministre de la santé et des services sociaux les informations nécessaires à une prise de décision sur la pratique des sages-femmes au Québec " (p. XIV) sur la base de recherches et de consultations, ces travaux, sous l'angle de la synthèse présentée dans cet ouvrage, permettent de jeter un éclairage sur la contribution spécifique des sages-femmes en périnatalité et sur l'impact de leur éventuelle intégration aux équipes de soins. Les débats sociaux sous-jacents à une telle publication, toujours en cours, concernent tout aussi bien les orientations politiques que le Québec devrait se donner en matière de périnatalité et l'évolution des pratiques sociosanitaires concernant les femmes, que la place des " pratiques alternatives en santé » et leur relation au système de santé officiel.

L'ouvrage comprend cinq sections. Dans la première, on présente la situation actuelle des pratiques obstétricales et les réponses offertes par le système de santé aux problèmes généraux relatifs à la périnatalité au Québec (mortalité périnatale, prématurité, interventionnisme médical, etc.). La section suivante présente la pratique des sages-femmes comme une manière de répondre aux besoins actuels de la société québécoise en matière de périnatalité. Entre autres, selon les auteures, la présence des sages-femmes permettrait une meilleure couverture des clientèles cibles, une amélioration de la continuité et de la qualité des soins, la réduction des naissances de bébés de faible poids et la réduction des césariennes. De plus, leur intégration donnerait enfin réponse aux revendications des femmes et couples exprimées depuis un bon moment quant à des services à la 
fois techniquement impeccables et humainement chaleureux (Saillant et al., 1987).

La section trois du volume constitue une exploration de la situation des sages-femmes telle qu'elle se présente au Québec (incluant un survol historique) et au Canada. Leur disparition est directement mise en relation avec la déshumanisation des soins en périnatalité, telle qu'elle fut observable surtout à partir de 1973. Le cadre juridique actuel y est aussi analysé. La quatrième section du document constitue une sorte de simulation des divers effets que pourrait avoir l'intégration des sages-femmes dans l'équipe de périnatalité (effets sur les effectifs sociosanitaires, sur les enjeux de santé publique, sur l'organisation des soins, sur les coûts). Enfin, la cinquième et dernière section inclut les recommandations du comité, dont plusieurs ont été concrétisées à la fin de 1989 ou au début de 1990, entre autres l'annonce de la loi autorisant l'implantation de projets pilotes permettant à des sages-femmes de pratiquer.

Cet ouvrage est un document d'importance, puisque les lecteurs et lectrices y trouveront l'essentiel des éléments d'information éclairant la situation des sages-femmes au Québec, sous forme d'une synthèse intelligente et présentée de façon très pédagogique. Plusieurs des annexes ont une valeur de référence très utile, entre autres une courte description de projets pilotes dans lesquels des sages-femmes ont été intégrées, des hypothèses sur le renouvellement des cadres juridiques (modifications à apporter à la loi médicale, notamment), ainsi que de l'information factuelle sur la situation des sages-femmes hors Canada. Le document montre donc les intentions progressistes de l'État dans le domaine, et constitue un dossier de qualité qu'apprécieront intervenants et intervenantes, chercheurs et chercheuses en périnatalité.

Les hypothèses retenues quant au cadre juridique (une loi spécifique pour les sages-femmes), au champ de pratique (déterminé en fonction de la situation existante dans les pays de la Communauté Économique Européenne), à la formation (qui devrait rencontrer les standards internationaux, exiger un niveau universitaire de $1^{\text {er }}$ cycle et comporter une solution pratique de transition pour les sages-femmes déjà formées) ou aux lieux de pratique (dans le réseau et à l'extérieur), sont des repères qui permettront d'évaluer l'avancement de ce dossier au cours des mois et années à venir. On doit cependant regretter le caractère flou des recommandations en ce qui a trait aux lieux de pratique, un aspect controversé de la pratique des sagesfemmes. Il est d'ailleurs à noter qu'il était prévu, à l'origine du projet de publication de cette série de dossiers, que l'un d'eux porterait sur les lieux de naissance; or, celui-ci n'a jamais vu le jour ! La situation des sages-femmes au Québec demeure donc, malgré l'acceptation au 
début de 1990 des projets pilotes par le ministre Marc-Yvan Côté, un dossier toujours explosif qui n'avance qu'à très petits pas, dont cet ouvrage rend fort bien compte.

\section{Adolescence et fertilité : une responsabilité person- nelle et sociale, par le Comité Enfance-Famille de la Division santé communautaire de l'Association des hôpitaux du Québec, Ministère de la Santé et des Services sociaux, Québec, 1989, 128 pages.}

Le rapport du Comité de travail Adolescence et fertilité : une responsabilité personnelle et sociale démontre que la grossesse à l'adolescence est un problème très important et dont on doit se préoccuper de façon prioritaire au Québec. Les données qui y sont présentées (chapitre IV) indiquent entre autres que plus d'une adolescente sur huit $(11,7 \%)$ nées en 1965-1966 ont accouché ou terminé une grossesse avant l'âge de 20 ans, et que $4,4 \%$ ont été dans la même situation avant d'avoir atteint l'âge de 18 ans. Le problème augmente au Québec, de façon plus prononcée chez les moins de 17 ans, alors qu'il est en régression ailleurs au Canada.

Les données relatives aux conditions sociales des mères adolescentes aident également à saisir l'importance de la situation. Bien que la grossesse à l'adolescence survienne dans toutes les classes sociales, la majorité de celles qui décident de poursuivre leur grossesse proviennent des milieux les plus défavorisés. La grossesse pendant l'adolescence entraîne des coûts très élevés (chapitre V). Si l'adolescente poursuit sa grossesse, elle donnera naissance à un enfant dont I'avenir est déjà sérieusement hypothéqué : le père géniteur s'étant le plus souvent éclipsé et ce, sous l'influence des parents de la jeune mère, cette dernière devra assumer seule des responsabilités qui deviennent vite très lourdes, à une période où elle est encore en situation de dépendance. La décision d'avoir recours à l'avortement 
est un choix très difficile à faire par les adolescentes concernées et, selon les auteurs et auteures de l'ouvrage, il ne doit pas être interprété comme une solution de facilité.

Au nombre des points forts, notons que les auteurs et auteures ont traité le problème de la grossesse en expliquant d'abord ce que signifie l'adolescence comme étape de développement et en situant l'expression de la sexualité à cette période de la vie. Les différents aspects du développement biologique, cognitif et psychosocial sont toutefois esquissés à grands traits au cours du premier chapitre, et le portrait type qu'on y fait de l'adolescent et de l'adolescente correspond à l'image la plus perturbée. Ce n'est qu'en fin de discussion que quelques nuances sont apportées : on indique que la recherche de l'identité, propre à l'adolescence, peut prendre une variété de formes d'expression et que les changements qui s'opèrent pendant cette période ne s'accompagnent pas nécessairement d'une crise. Les deuxième et troisième chapitres, respectivement consacrés à la sexualité et à la contraception à l'adolescence, sont aussi très brefs et les éléments qui y sont traités auraient pu l'être de façon plus approfondie.

Les chapitres VI, VII et VIII discutent des conditions qui amènent l'adolescente à devenir enceinte, de ses réactions et des choix qu'elle peut entrevoir. Cette section est traitée avec beaucoup de compréhension et de respect des émotions impliquées dans les décisions que l'adolescente doit prendre, à savoir poursuivre ou interrompre sa grossesse, et garder le bébé ou le donner en adoption. Le neuvième chapitre fait l'inventaire des services disponibles, aussi bien les services publics que l'aide pouvant provenir du réseau de support naturel. La conclusion de ce chapitre souligne l'inégalité régionale des services existants et la fragilité de plusieurs d'entre eux. Le dixième et dernier chapitre fait part des principes d'intervention retenus par le comité et des recommandations qu'il propose. Ces recommandations, au nombre de 54 , sont suivies d'un plan d'action qui place les recommandations en ordre de priorité.

Les recommandations, clairement formulées, sont supportées par les informations présentées dans les chapitres précédents. De façon globale, elles préconisent la mise sur pied d'un continuum de services, allant de la prévention primaire aux soins tertiaires, qui soient géographiquement et financièrement accessibles. C'est d'ailleurs en prenant connaissance des recommandations que le titre du livre prend tout son sens. Ces recommandations visent à la fois des interventions de nature à favoriser le développement de la responsabilité des adolescents et adolescentes face à leur fertilité et de nature à impliquer aussi les parents, les planificateurs et les intervenants. Le contenu des recommandations accuse cependant 
une certaine faiblesse au plan de la formation et de la recherche. D'une part, les recommandations concernant la formation des personnes impliquées dans l'implantation du programme de formation personnelle et sociale aux niveaux scolaires élémentaire et secondaire, auraient pu être plus énergiques, étant donné l'urgence d'agir dans ce domaine. D'autre part, il aurait été souhaitable que les recommandations portant sur la recherche insistent davantage sur la nécessité de faire avancer la connaissance des facteurs associés à la grossesse pendant l'adolescence, et de développer des stratégies d'intervention plus efficaces de même que des services mieux adaptés aux besoins des adolescents et adolescentes dans ce domaine.

II ne fait aucun doute que ce livre pourra être utile aux professionnels et professionnelles travaillant auprès de la population adolescente, qu'ils fassent partie du réseau de l'éducation, de la santé ou des services sociaux. Le langage utilisé étant facilement compréhensible, ce livre devrait également être mis à la disposition des parents. II pourrait ainsi constituer un premier effort éducatif à leur endroit.

La grossesse à l'adolescence constitue un véritable défi social. Maintenant que les faits ont été compilés et qu'il devient de plus en plus difficile de nier l'importance du problème, comment allons-nous y répondre en tant que société québécoise?

Michel O'Neill, professeur, École des sciences infirmières, Université Laval.

\section{Naître égaux et en santé, par C. COLIN et H. DeS- ROSIERS, Ministère de la Santé et des Services sociaux, Québec, 1989, 153 pages.}

En 153 pages, six chapitres et trois annexes, les auteures nous rappellent la brutale réalité que tous ne sont pas égaux au Québec en ce qui a trait à la périnatalité. D'entrée de jeu, elles soulignent que «[...] les taux obtenus en milieu défavorisé québécois se comparent à ceux des pays du Tiers Monde en Afrique, en Amérique latine ou en Océanie alors que les taux obtenus par les Québécoises les plus 
favorisées sont les mêmes que ceux obtenus par les pays scandinaves qui sont les meilleurs au monde»(p. xxiv).

Afin de mieux préciser l'objet de leur analyse, les auteures consacrent le premier chapitre de l'ouvrage à une excellente discussion de la pauvreté au Québec où elles passent en revue les principales définitions et mesures de celle-ci. Elles rappellent avec justesse que la pauvreté n'est pas qu'économique et que ses formes sont multiples, variant selon les groupes qu'elle affecte (enfants, jeunes et adolescents, chômeurs, chefs de famille monoparentale, immigrants, demandeurs de statut de réfugié, personnes âgées) et le degré de dénuement qu'elle occasionne (sous le seuil de faible revenu, sous le seuil de grande pauvreté). Elles montrent aussi ce qu'on tend souvent à ignorer : la pauvreté est en progression au Québec depuis 1980 et affecte $16 \%$ des individus, soit un million de personnes.

Le second chapitre fournit un portrait démographique de la grossesse en milieu défavorisé. Utilisant avec doigté et rigueur le peu d'information disponible sur le sujet, les auteures montrent qu'environ une grossesse sur six $(15 \%)$ au Québec serait vécue par des femmes bénéficiaires de l'aide sociale, dont une forte majorité se retrouve sous le seuil de l'extrême pauvreté. On passe ensuite en revue un certain nombre de caractéristiques des mères de milieu défavorisé; elles sont nettement moins scolarisées et plus souvent sans conjoint que les autres femmes et, même si. là encore les données fiables sont peu abondantes, il semble que la tendance observée un peu partout voulant que les femmes de milieu défavorisé aient plus d'enfants que celles des milieux aisés, se retrouve aussi au Québec.

Le chapitre suivant est probablement le plus intéressant du livre car, au-delà des chiffres, il s'attarde à cerner le vécu des femmes en milieu défavorisé qui enfantent, un vécu dont on ne sait généralement que peu de choses chez les intervenants et intervenantes, et qui est particulièrement crucial à comprendre si on veut arriver à aider vraiment cette clientèle. On y souligne entre autres que la grossesse est souvent un des seuls projets de vie que ces femmes, vivant dans des conditions extrêmement difficiles sur lesquelles elles n'ont que peu de contrôle, peuvent mener de manière autonome et valorisante. Ce chapitre fait aussi une présentation fort pertinente des habitudes de vie des femmes enceintes défavorisées, montrant là encore que si elles s'alimentent moins bien, fument davantage et font moins d'exercice que dans les milieux favorisés, cela s'explique et est cohérent avec leur univers de vie, un univers où on a spontanément tendance à se méfier des services officiels (de santé ou autres) qui souvent ne comprennent en rien leur situation. 
Le quatrième chapitre présente des données factuelles sur l'état de santé des femmes enceintes défavorisées et de leur nouveau-né. Après avoir discuté des principaux facteurs qui sont associés à une moins bonne santé maternelle et infantile, et présenté de manière imagée et particulièrement claire la kyrielle de taux utilisés par les chercheurs pour analyser la mortalité et la morbidité durant cette période, l'ouvrage confirme ce qu'on soupçonnait déjà : les nouveaunés de milieux défavorisés meurent davantage et sont plus malades que les autres, compte tenu du cumul des risques auxquels ils sont exposés à cause des conditions de vie précaires de leurs parents.

Le cinquième chapitre analyse l'état des services prénatals offerts en milieu défavorisé, en rappelant toutefois que ce sont des politiques économiques et sociales de réduction des inégalités, bien davantage que ces services, qui participeraient à affecter de manière significative la mortalité et la morbidité dans ces milieux. Quatre catégories de services sont passées en revue : les services médicaux de suivi de grossesse, les cours prénatals, le dépistage des grossesses "à haut risque " et les services particuliers de prévention prénatale en milieux défavorisés mis en œuvre au Québec et ailleurs. Ce chapitre s'appuie entre autres sur une excellente enquête (menée pour cet ouvrage) sur ce qui se passe en CLSC et DSC à ce propos, dont les principaux résultats sont livrés à l'annexe III. En gros, il est intéressant de noter qu'il y a dans notre système de soins (en particulier en CLSC) une bonne sensibilité à la nécessité de mettre en œuvre des services appropriés et au fait que le développement d'une attitude de travail différente de la part des intervenants et intervenantes est un des facteurs centraux à ce sujet. Les cas de réussites québécoises les mieux connus dans ce domaine (Dispensaire diététique de Montréal et projets OLO - oeufs, lait, oranges - en particulier) sont relatés dans ce chapitre et dans I'annexe III; qui plus est, la section sur les alternatives d'intervention, inspirées d'expériences étrangères, est particulièrement intéressante, donnant une série de suggestions très concrètes pour agir de manière efficace sur les grossesses en milieu défavorisé.

Le livre se termine par dix recommandations d'intervention à la fois au niveau des politiques gouvernementales et des actions régionales ou locales, de même que par une recommandation sur la formation et une autre sur la recherche. Dans ces deux derniers cas, il aurait été intéressant qu'on soit un peu plus explicite.

C'est peut-être un des seuls reproches qu'on peut adresser à cet ouvrage. En général, il est rédigé de manière simple et pédagogique tout en gardant constamment une excellente rigueur scientifique alimentée par une connaissance sophistiquée des publications internationales et des données québécoises existantes. On sent le parti 
pris des auteures envers une amélioration des services actuels dans des directions précises, par ailleurs supportées par la documentation internationale. On peut difficilement leur tenir rigueur de ce parti pris mais on peut toutefois se questionner sur la possibilité de mettre en œuvre de telles actions dans le contexte politique québécois des années 1990, où le néo-libéralisme incite davantage à rendre les pauvres responsables de leur sort et des moyens à prendre pour s'en sortir qu'à développer des politiques et interventions gouvernementales pour les aider à ce faire.

Denyse Leclerc, infirmière, Unité néonatale de I'Hôpital SaintFrançois d'Assise, Québec.

\section{La mortalité et la morbidité périnatales et infantiles, par le Comité de travail sur la mortalité et la morbidité périnatales, Ministère de la Santé et des Services sociaux, Québec, 1989, 251 pages.}

Totalisant 251 pages, ce livre met en évidence un problème de santé qui, malgré des améliorations sensibles au cours des derniers vingt ans, demeure prioritaire au Québec. Le premier chapitre illustre l'évolution des diverses mesures de la mortalité foeto-infantile et des caractéristiques de la natalité (mère et nouveau-né) qui ont une influence sur la mortalité et la morbidité périnatales. L'évolution de la mortalité maternelle, depuis 1976, y est également exposée. Le chapitre II décrit les conséquences de la mortalité et de la morbidité périnatales et infantiles. De son côté, le chapitre III présente une analyse de l'ensemble des facteurs de risque et des causes de ces mêmes problèmes. Ensuite, le chapitre IV fait ressortir certains éléments pour lesquels des interventions visant à diminuer l'impact des différents facteurs de risque sur le déroulement et l'issue de la grossesse ont été réalisées. Pour terminer, les auteurs proposent, dans un cinquième chapitre, un plan d'action qui tend à améliorer la santé et la qualité de vie des parents et nouveau-nés. 
On doit souligner, comme points particulièrement forts de cet ouvrage, qu'il relève les conséquences observées, en termes de morbidité chez l'enfant, d'une naissance prématurée ou de petit poids. De plus, on y aborde les répercussions d'une telle condition de naissance sur la relation parents-enfant et sur la dynamique familiale. Dans l'ensemble, ce livre fait ressortir la dimension multifactorielle du déroulement et de l'issue de la grossesse. On y mentionne en particulier les effets pathogènes du stress et la fonction immunitaire du réseau de soutien social sur celui-ci.

Concernant la prévention des grossesses à risque, les auteurs démontrent l'importance d'utiliser des stratégies d'intervention avant même la conception, ou très tôt au début de la grossesse. De plus, il est intéressant de noter que parmi les éléments pour lesquels des interventions ont été réalisées, on souligne l'impact des facteurs intrapsychiques : ceux-ci sont susceptibles d'entraver de façon significative l'évolution normale de la grossesse et du processus d'attachement à son enfant. Dans l'élaboration du plan d'action, on propose, entre autres, la participation active des parents aux décisions relatives à l'évolution de la grossesse et la reconnaissance de la sage-femme comme alternative au "suivi médical traditionnel ".

Certains points de l'ouvrage sont plus faibles cependant. Bien que l'on cite quelques facteurs pouvant expliquer les problèmes de santé observés chez l'enfant né prématurément ou de petit poids de naissance, on a omis de faire ressortir ceux liés aux conditions de vie néonatale de ces enfants. Ces conditions se répercutent en particulier au niveau de la psychogénèse et, potentiellement, peuvent être à la base des déficits identifiés, puisqu'elles impliquent souffrances physiques et carence affective lors de la phase la plus précoce de la vie. De plus, I'analyse de l'ensemble des facteurs de risque susceptibles d'affecter le déroulement et l'issue de la grossesse ne tient pas compte des sentiments de la mère vis-à-vis sa propre grossesse et son foetus. Plusieurs études ont montré qu'une attitude maternelle négative (consciente ou inconsciente) peut s'exprimer par une tendance à adopter des comportements à risque et que, non seulement l'issue de la grossesse peut en être affectée, mais certains problèmes de santé des enfants portés dans un tel climat en dépendent (Ferreire, 1965; Guitouni, 1982; Sontag, 1966; Tomatis, 1980; Verny, 1981; Weill et Tupper, 1960).

Portrait juste de la situation québécoise, cet ouvrage constitue un précieux outil de référence pour les professionnelles et professionnels de la santé désireux d'améliorer la qualité de vie des parents et des nouveau-nés. Axé sur une approche globale et préventive, il fournit l'information nécessaire à la préparation d'un enseignement préventif ainsi qu'au choix de stratégies d'action pertinentes aux 
diverses populations cibles. La mise en œuvre de ces interventions stratégiques est cruciale car, faut-il se le rappeler, les nouveau-nés d'aujourd'hui formeront la société d'adultes de demain !

Linda Lepage, professeure, École des sciences infirmières, Université Laval.

Vivre avec un nourrisson, par le Comité EnfanceFamille de la Division santé communautaire de I'Association des hôpitaux du Québec, Ministère de la Santé et des Services sociaux, Québec, 1989, 292 pages.

Vivre avec un nourrisson est un avis sur la période post-natale qui devrait intéresser tant les gestionnaires que les planificateurs ou les intervenantes et intervenants en périnatalité préoccupés par le soutien aux parents et le développement de l'enfant. II a été préparé par un sous-groupe du Comité Enfance-Famille de la Division santé communautaire de l'Association des hôpitaux du Québec.

L'avis est subdivisé en huit chapitres. Le premier présente les principales dimensions de la situation démographique québécoise liée à la périnatalité, alors que le deuxième chapitre traite des besoins, des problèmes de santé du nourrisson et de leur évolution dans le contexte de ce qu'on appelle les "nouvelles morbidités ": abus et négligence, retards de développement, troubles de comportement. Les auteures insistent sur l'origine multifactorielle de ces derniers problèmes, leur persistance dans le temps, leur impact sur la famille et leur variation selon le milieu socio-économique. Le chapitre III décrit les stress auxquels sont confrontés les nouveaux parents dans l'apprentissage de leur rôle et les ajustements qu'ils doivent faire pour répondre aux besoins de leur enfant mais aussi à leurs besoins personnels ou à ceux de leur couple et de leur famille.

Les facteurs facilitant ou entravant cet ajustement parental sont d'abord traités lors d'une situation d'adaptation régulière et ensuite repris, dans le chapitre suivant, en tenant compte cette fois de 
situations particulières : adolescence, monoparentalité, toxicomanie, conflits conjugaux, milieu défavorisé, situation de réfugié ou d'autochtone. La "mal adaptation» parentale sous différentes formes (mauvais traitements et dépression post-natale) fait l'objet du cinquième chapitre. Ces deux chapitres présentent un état de la situation et des facteurs qui l'influencent, ainsi que les interventions actuelles et celles à développer.

Le sixième chapitre est consacré pour sa part aux services en période post-natale. Une description des services basée sur ceux offerts dans trois régions du Québec (urbaine, périphérique, éloignée) est d'abord présentée. Cette première partie du chapitre est suivie d'un examen des principaux problèmes rencontrés par ces services, problèmes mis en lien avec la situation d'adaptation des parents. Ce chapitre se termine par une présentation des résultats d'études évaluatives de certaines interventions préventives. Dans le chapitre VII, des parents s'expriment sur ce qu'ils vivent au cours de cette période $d^{\prime}$ adaptation et suggèrent quelques pistes d'orientation des services. Finalement, le dernier chapitre propose une série de recommandations découlant des analyses et réflexions précédentes, de même qu'une nouvelle orientation des services post-natals. Certaines recommandations touchent également la formation et la recherche et sont toutes assorties d'une identification d'actions prioritaires.

Cet avis propose un renouvellement de perspective en abordant l'adaptation parentale et familiale à la naissance d'un enfant comme un processus qui s'étend de la grossesse jusqu'à la fin de la première année de vie de l'enfant. De plus, en traitant à partir d'une approche écologique les facteurs influençant cette adaptation, à savoir la recherche d'un nouvel équilibre dans la dynamique familiale, les auteures dépassent largement les caractéristiques personnelles de chacun des individus qui composent la famille. Elles font en effet ressortir les caractéristiques structurelles et fonctionnelles de la famille affectant l'adaptation et les placent en interrelation avec les contextes sociaux et physiques dans lesquels évolue cette famille. La qualité des interactions parents-enfant et le développement de l'enfant se conçoivent alors non seulement comme une responsabilité familiale, mais aussi comme une responsabilité de la communauté de vie de la famille et même de la société tout entière.

Les auteures de cet avis annoncent dès l'introduction qu'elles se sont attardées au développement de services préventifs et qu'elles ont mis l'accent sur la prévention primaire. Dans ce sens, et à partir de la nouvelle perspective proposée, elles dégagent une vision différente des services offerts et à offrir, tant dans une situation régulière que dans un contexte particulier d'adaptation parentale ou dans une 
situation où les parents sont mal adaptés. Peu importe la situation, la plupart des parents sont perçus comme ayant les ressources qui leur permettent d'assurer le développement de leur enfant, en autant qu'ils bénéficient du soutien dont ils ont besoin dans un contexte facilitant l'actualisation de leur rôle parental. Le but de toute intervention préventive est donc vu comme l'apport de ce soutien, via une mobilisation des ressources des parents et de celles de leur milieu de vie, mais également par une amélioration de leurs conditions de vie dans leur quartier, au travail et dans la société en général. Selon les nombreux intervenants et parents consultés, la complexité et l'efficacité des interventions préventives exigent un travail en complémentarité, une concertation et une intégration des acquis des acteurs concernés : les différents regroupements sociaux (groupes et organismes communautaires, groupes de parents, syndicats, etc.), les intervenants de différentes professions, les institutions et les différents paliers de planification (local, régional, provincial et national).

Ces divers points sont abordés avec clarté et précision; ils sont appuyés par une excellente recension des publications lorsque cela est pertinent. La liste des références, développée à la fin de chaque chapitre, permettra aux lectrices et lecteurs moins familiers ou plus intéressés par l'un ou l'autre des éléments du contenu, de se documenter facilement. La table des matières, très spécifique, facilite le repérage rapide de chacun des sujets traités.

Soulignons toutefois que même si l'ensemble du document est très cohérent, un décalage important est observable entre le corps du texte et les objectifs d'intervention du dernier chapitre. Tout au long de l'ouvrage, les auteures insistent sur la quasi-absence de données scientifiques rigoureuses permettant de brosser un portrait fiable de la situation québécoise, en particulier sur des sujets tels les mauvais traitements, l'alimentation du nourrisson ou l'immunisation. Qui plus est, elles recommandent, quant à la recherche, de mieux documenter ces sujets afin « [...] de fixer des objectifs réalistes de diminution des problèmes majeurs ressentis» (p. 273). On s'étonne donc de retrouver dans les recommandations des phrases telles que : "Réduire de $20 \%$ le taux d'abus et de négligence envers les enfants d'ici I'an 2000 ", ou encore "Retarder l'introduction des solides entre 4 et 6 mois chez $80 \%$ des nourrissons" (p. 257). Comment peut-on en effet diminuer de $X \%$ des problèmes dont on ne connaît même pas l'ampleur actuelle?

Il est bien évident que les multiples particularités des diverses expériences familiales d'adaptation post-natale, de même que chacune des interventions actuelles au Québec, n'ont pu être traitées dans cette publication. Cependant elle constitue, de l'avis même des 
auteures, " un premier bilan, le plus exhaustif et le plus révélateur possible, de la situation en période post-natale au Québec » (p. 7). Nous ne pouvons qu'applaudir à leur remarquable travail en espérant qu'il influencera les orientations politiques québécoises dans le domaine périnatal.

\section{Conclusion}

Si l'on pose un regard d'ensemble sur le contenu des cinq livres qui composent la série La périnatalité au Québec, on en arrive sans l'ombre d'un doute à la conclusion qu'il s'agit là d'un excellent ensemble d'ouvrages. Dans tous les cas, les équipes réunies constituaient une sorte de "Who's who " québécois face au sujet traité. Cela a donc permis que les connaissances recueillies et analysées se situent à la fine pointe du savoir, que souvent des comparaisons pertinentes soient établies entre le Québec et le reste du Canada ou même avec d'autres pays, que la rigueur scientifique de l'entreprise soit démontrée hors de tout doute et que, finalement, des problématiques intéressantes et des recommandations d'interventions généralement très appropriées soient apportées.

Ce qui est particulièrement remarquable de cette série, c'est le mode de présentation adopté. Même quand des sujets très techniques sont abordés, le langage demeure accessible, les points essentiels sont mis en encart et une présentation visuelle soignée, appuyée par des tableaux et des graphiques fort clairs, permet d'aller tout de suite à l'essentiel. Les bibliographies, à la fin de chaque ouvrage, sont des sources de référence de tout premier ordre pour qui veut poursuivre étude ou réflexion. Même si on ne peut qualifier cette série de " grand public ", les différents groupes d'intervenants et intervenantes en périnatalité $s^{\prime} y$ retrouveront avec grande facilité; les étudiants et étudiantes en sciences sociales ou de la santé y trouveront un intérêt certain, tout comme un public général averti.

Là où l'on peut cependant questionner la pertinence de ces cinq ouvrages, c'est du point de vue de l'avancement politique du dossier de la périnatalité au Québec. En effet ces cinq études, malgré leurs qualités, reprennent en les vulgarisant et en les rendant accessibles à un plus large public des informations qui, pour la plupart, étaient déjà connues et publiées dans des études ou documents spécialisés. Une fois de plus, l'ensemble de l'opération sent la diversion : quand la pression politique monte, comme après la publication du projet de politique en périnatalité de 1985, plutôt que d'agir, le MSSS crée non pas un, mais cinq comités d'experts, ce qui permet de gagner du 
temps et de repousser à plus tard les délicates prises de décision dans l'univers de la périnatalité.

La série d'ouvrages analysés plus haut vient renforcer la nécessité, déjà bien campée dans le projet de politique de 1985, de la prévention et de la mise en œuvre de moyens respectant davantage la population et coûtant moins cher que les modes actuels de dispensation des services périnatals. En ce sens, cette série vient ajouter quelques briques à l'édifice qui se construit depuis vingt ans pour proposer des changements significatifs aux soins et services périnatals tels que dispensés présentement au Québec. Cependant, tant que la volonté du gouvernement du Québec d'affronter le pouvoir médical sur ce terrain de manière tenace et définitive ne sera pas claire, I'utilité réelle de publications comme celles analysées ici demeure somme toute mitigée.

\section{Références}

BLANCHET, M. (1979). " L'influence des politiques gouvernementales sur la santé maternelle ", La vie médicale au Canada français, vol. 8, $\mathrm{n}^{\circ} 1$ : 24-47.

Colin, C. et H. Desrosiers (1989). Naître égaux et en santé. Québec : Ministère de la Santé et des Services sociaux, 153 pages ( $n^{\circ} 3$ de la collection La périnatalité au Québec).

Comité de travail sur la pratique des sages-femmes au Québec (1989). La pratique des sages-femmes. Québec: Ministère de la Santé et des Services sociaux, 155 pages $\left(\mathrm{n}^{\circ} 1\right.$ de la collection La périnatalité $a u$ Québec).

Comité Enfance-Famille de la Division santé communautaire de l'Association des hôpitaux du Québec (1989). Adolescence et fertilité : une responsabilité personnelle et sociale. Québec : Ministère de la Santé et des Services sociaux, 128 pages $\left(\mathrm{n}^{\circ} 2\right.$ de la collection La périnatalité au Québec).

Comité de travail sur la mortalité et la morbidité périnatales (1989). La mortalité et la morbidité périnatales et infantiles. Québec: Ministère de la Santé et des Services sociaux, 251 pages $\left(n^{\circ} 4\right.$ de la collection La périnatalité au Québec).

Comité Enfance-Famille de la Division santé communautaire de l'Association des hôpitaux du Québec (1989). Vivre avec un nourrisson. Québec : Ministère de la Santé et des Services sociaux, 292 pages $\left(n^{\circ} 5\right.$ de la collection La périnatalité au Québec).

COURNOYER, Monique (1987). "L'accouchement, d'hier à demain : les femmes cries de la Baie-James ": 99-125, dans Francine Saillant et Michel O'Neill (éd.), Accoucher autrement : repères historiques, sociaux et culturels sur la grossesse et l'accouchement au Québec. Montréal : Éditions SaintMartin.

DE KONINCK, Maria (1981). "Accoucher ou se faire accoucher: rapport synthèse des onze colloques régionaux ", Bulletin de l'ASPQ, vol. 5, $\mathrm{n}^{\circ} 1$ : 5-16. 
De Koninck, Maria, Francine SaILlant et Louise DUNIGAN (1981). Essai sur la santé des femmes. Éditeur officiel du Québec.

DUfOuR, Rose (1987). "Accoucher dans un iglou " : 73-99, dans Francine Saillant et Michel $\mathrm{O}^{\prime}$ Neill (éd.), Accoucher autrement: repères historiques, sociaux et culturels sur la grossesse et l'accouchement au Québec. Montréal : Éditions Saint-Martin.

FERREIRE, A.J. (1965). "Emotional factors in prenatal environment ", The Journal of Nervous and Mental Disease, vol. 141, $\mathrm{n}^{\circ} 1: 108-118$.

GuitounI, M. (1982). "La genèse de la dépression et la possibilité d'une approche préventive ", Psychologie préventive, vol. 1, $\mathrm{n}^{\circ} 2: 15-30$.

LAFORCE, Hélène (1987a). "L'accouchement traditionnel : d'hier à aujourd'hui »: 31-39, dans Francine Saillant et Michel O'Neill (éd.), Accoucher autrement : repères historiques, sociaux et culturels sur la grossesse et l'accouchement au Québec. Montréal : Éditions Saint-Martin.

LAFORCE, Hélène (1987b). "Les grandes étapes de l'élimination des sagesfemmes au Québec du $17^{\mathrm{e}}$ au $20^{\mathrm{e}}$ siècle »: 163-181, dans Francine Saillant et Michel O'Neill (éd.), Accoucher autrement: repères historiques, sociaux et culturels sur la grossesse et l'accouchement au Québec. Montréal : Éditions Saint-Martin.

LaurendeAu, France (1987). "La médicalisation de I'accouchement » : 125-163, dans Francine Saillant et Michel O'Neill (éd.), Accoucher autrement : repères historiques, sociaux et culturels sur la grossesse et l'accouchement au Québec. Montréal : Éditions Saint-Martin.

Ministère des Affaires sociales du Québec (1985). Projet de politique en périnatalité.

ROUTHIER, Marie-Josée (1987). «Que sont devenues les sages-femmes d'antan ? L'accouchement chez les femmes attikamèques de Manouane ": 39-73, dans Francine Saillant et Michel O'Neill (éd.), Accoucher autrement: repères historiques, sociaux et culture/s sur la grossesse et l'accouchement au Québec. Montréal : Éditions Saint-Martin.

SAILLANT, Francine (1985). "Le mouvement pour la santé des femmes »: 743763, dans Jacques Dufresne et al., (éd.), Traité d'anthropologie médicale. Québec et Lyon : Presses de I'Université du Québec; IQRC et Presses Universitaires de Lyon.

SaIllant, Francine, Michel O'NeILl et Danièle Desjardins (1987). "Entre le cœur et la raison : portrait de la clientèle d'une nouvelle sage-femme québécoise ": 295-317, dans Francine Saillant et Michel O'Neill (éd.), Accoucher autrement: repères historiques, sociaux et culturels sur la grossesse et l'accouchement au Québec. Montréal : Éditions Saint-Martin.

SAVARD, Suzanne (1987). "Accoucher à l'hôpital : où en sommes-nous aujourd'hui ? " : 279-295, dans Francine Saillant et Michel O'Neill (éd.), Accoucher autrement: repères historiques, sociaux et culturels sur la grossesse et l'accouchement au Québec. Montréal : Éditions Saint-Martin.

SONTAG, L. (1966). "Implications of fetal behavior and environment for adult personalities ", Annals of New York Academy of Sciences, vol. 134 : 782786.

TOMATIS, A.A. (1981). La nuit utérine. Paris : Stock.

VERNY, Th. (1981). The secret life of the unborn child. Don Mills : Collins.

WeILL, R.J. et C. TUPPER (1960). "Personality, life-situation, and communication : a study of habitual abortion ", Psychosomatic Medicine, vol. 22, $\mathrm{n}^{\circ} 6: 448-455$. 